\title{
An Isomonodromy Interpretation of the Hypergeometric Solution of the Elliptic Painlevé Equation (and Generalizations)^
}

Eric M. RAINS

Department of Mathematics, California Institute of Technology, 1200 E. California Boulevard, Pasadena, CA 91125, USA

E-mail: rains@caltech.edu

Received April 25, 2011, in final form September 06, 2011; Published online September 09, 2011 http://dx.doi.org/10.3842/SIGMA.2011.088

\begin{abstract}
We construct a family of second-order linear difference equations parametrized by the hypergeometric solution of the elliptic Painlevé equation (or higher-order analogues), and admitting a large family of monodromy-preserving deformations. The solutions are certain semiclassical biorthogonal functions (and their Cauchy transforms), biorthogonal with respect to higher-order analogues of Spiridonov's elliptic beta integral.
\end{abstract}

Key words: isomonodromy; hypergeometric; Painlevé; biorthogonal functions

2010 Mathematics Subject Classification: 33E17; 34M55; 39A13

\section{Introduction}

In [21], Sakai introduced an elliptic analogue of the Painlevé equations, including all of the known discrete (and continuous) Painlevé equations as special cases. Unfortunately, although Sakai's construction is quite natural and geometric, it does not reflect the most important role of the ordinary Painlevé transcendents, namely as parameters controlling monodromy-preserving deformations.

As with the ordinary Painlevé equations, the elliptic Painlevé equation admits a special class of "hypergeometric" solutions $[12,18]$ that in the most general case can be expressed via $n$ dimensional contour integrals with integrands expressed in terms of elliptic Gamma functions. It is thus natural, as a first step in constructing an isomonodromy interpretation of the elliptic Painlevé equation, to attempt to understand that interpretation in the hypergeometric case (and thus gain insight to the general case). Note that we want to understand the hypergeometric case for all $n \geq 1$, to avoid the possibility that the small $n$ cases might differ from the general Painlevé case in some qualitatively significant way. (For instance, [13] considers the isomonodromy interpretation of the usual ${ }_{2} F_{1}$ (corresponding to $n=1$ in our setting), but this is simplified greatly from the general Painlevé VI case by the fact that not only the monodromy but the equation itself can be taken to be triangular.)

In the present work, we do precisely that: associated to each elliptic hypergeometric solution of the elliptic Painlevé equation, we construct a corresponding second-order linear difference equation that admits a family of discrete "monodromy-preserving" deformations. (In fact, the construction works equally well for higher-order analogues of the relevant elliptic hypergeometric integrals, which should correspond to special solutions of "elliptic Garnier equations".) The construction is based on an analogue of the approach in [15, 11]. There, a linear differential

${ }^{\star}$ This paper is a contribution to the Special Issue "Relationship of Orthogonal Polynomials and Special Functions with Quantum Groups and Integrable Systems". The full collection is available at http://www.emis.de/journals/SIGMA/OPSF.html 
equation deformed by the hypergeometric case of the Painlevé VI equation is constructed as a differential equation satisfied by a family of "semiclassical" (bi-)orthogonal polynomials. Our construction is much the same, although there are several technical issues to overcome.

The first such issue is, simply put, to understand precisely what it means for a deformation of an elliptic difference equation to preserve monodromy, or even what the monodromy of an elliptic difference equation is. While we give only a partial answer to this question, we do define (in Section 2 below; note that many of the considerations there turn out to have been anticipated by Etingof in [8]) a weakened form of monodromy that, while somewhat weaker than the analogous notions at the $q$-difference [9] and lower [4] levels, is still strong enough to give a reasonably rigid notion of isomonodromy deformation. Indeed, two elliptic difference equations have the same weak monodromy iff the corresponding difference modules (see [17]) are isomorphic; the same holds for ordinary difference equations, even relative to the stronger notion of monodromy [5]. The key observation is that a fundamental matrix for a $p$-elliptic $q$-difference equation is also a fundamental matrix for a $q$-elliptic $p$-difference equation; this latter equation (up to a certain equivalence relation) plays the role of the monodromy. (The result is similar to the notion of monodromy introduced by Krichever in [14]; while our notion is weakened by an equivalence relation, it avoids any assumptions of genericity.)

In Section 3, we develop the theory of semiclassical elliptic biorthogonal functions, functions biorthogonal with respect to a density generalizing Spiridonov's elliptic beta integral [23] by adding $m$ additional pairs of parameters. The key observation is that such functions can be constructed as higher-order elliptic Selberg integrals of a special form; in addition, their "Cauchy transforms" can also be so written. This gives rise to several nice relations between these functions, which we describe. Most important for our purposes is their behavior under $p$-shifts; the biorthogonal functions themselves are $p$-elliptic, but if we include the Cauchy transforms, the overall action is triangular. We can thus construct from these functions a $2 \times 2$ matrix which satisfies a triangular $q$-elliptic $p$-difference equation, analogous to the Riemann-Hilbert problem associated to orthogonal polynomials ([10, § 3.4]; see also [6] for a general exposition). By the theory of Section 2, this immediately gives rise to a $p$-elliptic $q$-difference equation, and symmetries of the $p$-difference equation induce monodromy-preserving deformations of the $q$-difference equation.

Finally, in Section 4, we compute this difference equation and the associated deformations. Although we cannot give a closed form expression for the difference equation, we are able at least to determine precisely where the difference equation is singular, and at each such point, compute the value (or residue, as appropriate) of the shift matrix. Together with the fact that the coefficients are meromorphic $p$-theta functions, this data suffices to (over)determine the shift matrix.

In a followup paper [3], with Arinkin and Borodin, we will complete the isomonodromy interpretation of the elliptic Painlevé equation by applying the ideas of [2] to show that any difference equation having the same structure as the ones constructed below admits a corresponding family of monodromy-preserving deformations, and moreover that (when $m=1$ ) Sakai's rational surface can be recovered as a moduli space of such difference equations. A rather different geometric approach to such an interpretation (via a Lax pair) for the $m=1$ case has been given in $[26]$.

\section{Notation}

The elliptic Gamma function [20] is defined for complex numbers $p, q, z$ with $|p|,|q|<1, z \neq 0$, by

$$
\Gamma_{p, q}(z):=\prod_{0 \leq i, j} \frac{1-p^{i+1} q^{j+1} / z}{1-p^{i} q^{j} z}
$$


and satisfies the reflection relation

$$
\Gamma_{p, q}(p q / z)=\Gamma_{p, q}(z)^{-1}
$$

as well as the shift relations

$$
\Gamma_{p, q}(p z)=\theta_{q}(z) \Gamma_{p, q}(z), \quad \Gamma_{p, q}(q z)=\theta_{p}(z) \Gamma_{p, q}(z),
$$

where the function

$$
\theta_{p}(z):=\prod_{0 \leq i}\left(1-p^{i+1} / z\right)\left(1-p^{i} z\right)
$$

satisfies

$$
\theta_{p}(z)=-z \theta_{p}(1 / z)=\theta_{p}(p / z)
$$

so that

$$
\Gamma_{p, q}(p q z) \Gamma_{p, q}(z)=-z^{-1} \Gamma_{p, q}(p z) \Gamma_{p, q}(q z)
$$

By convention, multiple arguments to a Gamma or theta function represent a product; thus, for instance

$$
\Gamma_{p, q}\left(u_{0} z^{ \pm 1}\right)=\Gamma_{p, q}\left(u_{0} z\right) \Gamma_{p, q}\left(u_{0} / z\right) .
$$

We will also make brief use of the third-order elliptic Gamma function

$$
\Gamma_{p, q, t}^{+}(x):=\prod_{0 \leq i, j, k}\left(1-p^{i} q^{j} t^{k} x\right)\left(1-p^{i+1} q^{j+1} t^{k+1} / x\right),
$$

which satisfies

$$
\Gamma_{p, q, t}^{+}(t x)=\Gamma_{p, q}(x) \Gamma_{p, q, t}^{+}(x), \quad \Gamma_{p, q, t}^{+}(p q t / x)=\Gamma_{p, q, t}^{+}(x) ;
$$

for our purposes, this appears only as a normalization factor relating the order 1 elliptic Selberg integral to the hypergeometric tau function for elliptic Painlevé.

\section{$2 \quad$ Elliptic difference equations}

Let $p$ be a complex number with $|p|<1$. A (meromorphic) $p$-theta function of multiplier $\alpha z^{k}$ is a meromorphic function $f(z)$ on $\mathbb{C}^{*}:=\mathbb{C} \backslash\{0\}$ with the periodicity property $f(p z)=\alpha z^{k} f(z)$. (To justify this definition, observe that the composition $f(\exp (2 \pi \sqrt{-1} t))$ is meromorphic on $\mathbb{C}$, periodic with period 1, and quasi-periodic with period $\log (p) / 2 \pi \sqrt{-1}$; in other words, it is a theta function in the usual sense.) The canonical example of such a function is $\theta_{p}(z)$, a holomorphic $p$ theta function with multiplier $-z^{-1}$; indeed, any holomorphic $p$-theta function can be written as a product of functions $\theta_{p}(u z)$, and any meromorphic $p$-theta function as a ratio of such products. In the special case of multiplier 1 , the function is called $p$-elliptic, for similar reasons. By standard convention, a $p$-theta function, if not explicitly allowed to be meromorphic, is holomorphic; however, $p$-elliptic functions are always allowed to be meromorphic (since a holomorphic $p$ elliptic function is constant).

Let $q$ be another complex number with $|q|<1$, such that $p^{\mathbb{Z}} \cap q^{\mathbb{Z}}=\varnothing$. 
Definition 2.1. A $p$-theta $q$-difference equation of multiplier $\mu(z)=\alpha z^{k}$ is an equation of the form

$$
v(q z)=A(z) v(z),
$$

where $A(z)$ is a nonsingular meromorphic matrix (a square matrix, each coefficient of which is meromorphic on $\mathbb{C}^{*}$, and the determinant of which is not identically 0 ), called the shift matrix of the equation, such that

$$
A(p z)=\mu(z) A(z),
$$

so in particular the coefficients of $A$ are meromorphic $p$-theta functions of multiplier $\mu(z)$. Similarly, a $p$-elliptic $q$-difference equation is a $p$-theta $q$-difference equation of multiplier 1.

We will refer to the dimension of the matrix $A$ as the order of the corresponding difference equation. We note the following fact about nonsingular meromorphic matrices.

Proposition 2.2. Let $M(z)$ be a nonsingular meromorphic matrix. Then $M(z)^{-1}$ is also a nonsingular meromorphic matrix, and if the coefficients of $M(z)$ are meromorphic p-theta functions of multiplier $\mu(z)$, then those of $M(z)^{-1}$ are meromorphic $p$-theta functions of multiplier $\mu(z)^{-1}$.

Proof. Indeed, the coefficients of the adjoint matrix $\operatorname{det}(M(z)) M(z)^{-1}$ are minors of $M(z)$, and thus, as polynomials in meromorphic functions, are meromorphic; this continues to hold after multiplying by the meromorphic function $\operatorname{det}(M(z))^{-1}$. For the second claim, if

$$
M(p z)=\mu(z) M(z)
$$

then

$$
M(p z)^{-1}=\mu(z)^{-1} M(z)^{-1} .
$$

Definition 2.3. Let $v(q z)=A(z) v(z)$ be a $p$-theta $q$-difference equation. A meromorphic fundamental matrix for this equation is a nonsingular meromorphic matrix $M(z)$ satisfying

$$
M(q z)=A(z) M(z) .
$$

It follows from a theorem of Praagman [16, Theorem 3] that for any nonsingular meromorphic matrix $A(z)$, there exists a nonsingular meromorphic matrix $M(z)$ satisfying $M(q z)=A(z) M(z)$ (this is the special case of the theorem in which the discontinuous group acting on $\mathbb{C P}^{1}$ is that generated by multiplication by $q$ ). In particular, any $p$-theta $q$-difference equation admits a meromorphic fundamental matrix. In the case of a first order equation, we can explicitly construct such a matrix.

Proposition 2.4. Any first order p-theta q-difference equation admits a meromorphic fundamental matrix.

Proof. For any nonzero meromorphic $p$-theta function $a(z)$, we need to construct a nonzero meromorphic function $f(z)$ such that

$$
f(q z)=a(z) f(z) .
$$

Since $a(z)$ can be factored into functions $\theta_{p}(u z)$, it suffices to consider the case $a(z)=\theta_{p}(u z)$, with meromorphic solution

$$
f(z)=\Gamma_{p, q}(u z) ;
$$

this includes the case $a(z)=b z^{k}$ by writing

$$
b z^{k}=\frac{\theta_{p}(-b z) \theta_{p}(-p z)^{k-1}}{\theta_{p}(-b p z) \theta_{p}(-z)^{k-1}} .
$$


We note in particular that, since the elliptic Gamma function is symmetrical in $p$ and $q$, the solution thus obtained for a first order $p$-theta $q$-difference equation also satisfies a $q$-theta $p$-difference equation. This is quite typical, and in fact we have the following result.

Lemma 2.5. Let $v(q z)=A(z) v(z)$ be a p-theta q-difference equation of multiplier $\mu(z)$, and let $M(z)$ be a meromorphic fundamental matrix for this equation. Then there exists a (unique) $q$-theta $p$-difference equation of multiplier $\mu(z)$ for which $M(z)^{t}$ is a fundamental matrix.

Proof. An equation $w(p z)=C(z) w(z)$ with fundamental matrix $M(z)^{t}$ satisfies

$$
M(p z)^{t}=C(z) M(z)^{t}
$$

and thus, since $M(z)$ is nonsingular, we can compute

$$
C(z)=M(p z)^{t} M(z)^{-t}
$$

(Here $M^{-t}$ denotes the inverse of the transpose of $M$.) This matrix is meromorphic, and satisfies

$$
C(q z)=M(p q z)^{t} M(q z)^{-t}=M(p z) A(q z)^{t} A(z)^{-t} M(z)^{-t}=\mu(z) C(z) .
$$

By symmetry, we obtain the following result.

Theorem 2.6. Let $M(z)$ be a nonsingular meromorphic matrix. Then the following are equivalent:

(1) $M(z)$ is a meromorphic fundamental matrix for some p-theta q-difference equation;

(2) $M(z)^{t}$ is a meromorphic fundamental matrix for some q-theta p-difference equation;

$\left(1^{\prime}\right) M(z)^{-t}$ is a meromorphic fundamental matrix for some -theta $q$-difference equation;

$\left(2^{\prime}\right) M(z)^{-1}$ is a meromorphic fundamental matrix for some q-theta $p$-difference equation,

as are the corresponding statements with "some" replaced by "a unique". Furthermore, if the above conditions hold, the multipliers of the difference equations of (1) and (2) agree, and are inverse to those of $\left(1^{\prime}\right)$ and $\left(2^{\prime}\right)$.

Remark 2.7. In the elliptic case, the above observations were made by Etingof [8], who also noted that the associated $q$-elliptic $p$-difference equation can be thought of as the monodromy of $M$.

Given a $p$-theta $q$-difference equation, the corresponding meromorphic fundamental matrix is by no means unique, and thus we obtain a whole family of related $q$-theta $p$-difference equations. There is, however, a natural equivalence relation on $q$-theta $p$-difference equations such that any $p$-theta $q$-difference equation gives rise to a well-defined equivalence class. First, we need to understand the extent to which the fundamental matrix fails to be unique.

Lemma 2.8. Let $M(z)$ and $M^{\prime}(z)$ be fundamental matrices for the same p-theta q-difference equation $v(q z)=A(z) v(z)$. Then

$$
M^{\prime}(z)=M(z) D(z)^{t}
$$

for some nonsingular meromorphic matrix $D(z)$ with q-elliptic coefficients. 
Proof. Certainly, there is a unique meromorphic matrix $D(z)$ with $M^{\prime}(z)=M(z) D(z)^{t}$, and comparing determinants shows it to be nonsingular. It thus remains to show that $D(z)$ has $q$-elliptic coefficients, or equivalently that $D(q z)=D(z)$. As in the proof of Lemma 2.5, we can write

$$
A(z)=M(q z) M(z)^{-1}=M^{\prime}(q z) M^{\prime}(z)^{-1},
$$

and thus

$$
D(q z)^{t} D(z)^{-t}=M(q z)^{-1} M^{\prime}(q z) M^{\prime}(z)^{-1} M(z)=1,
$$

as required.

Theorem 2.9. Define an equivalence relation on q-theta p-difference equations by saying

$$
[v(p z)=C(z) v(z)] \cong\left[v(p z)=C^{\prime}(z) v(z)\right]
$$

iff there exists a nonsingular q-elliptic matrix $D(z)$ such that

$$
C^{\prime}(z) D(z)=D(p z) C(z) \text {. }
$$

Then the set of q-theta $p$-difference equations associated to a given p-theta q-difference equation is an equivalence class.

Proof. Let $M(z)$ be a meromorphic fundamental matrix for the $p$-theta $q$-difference equation $v(q z)=A(z) v(z)$, and associated $q$-theta $p$-difference equation $w(p z)=C(z) w(z)$. If $M^{\prime}(z)$ is another meromorphic fundamental matrix for the $q$-difference equation, with associated $p$ difference equation $w(p z)=C^{\prime}(z) w(z)$, then

$$
M^{\prime}(z)=M(z) D(z)^{t},
$$

and thus

$$
C^{\prime}(z)=M^{\prime}(p z)^{t} M^{\prime}(z)^{-t}=D(p z) M(p z)^{t} M(z)^{-t} D(z)^{-1}=D(p z) C(z) D(z)^{-1} .
$$

Conversely, if

$$
C^{\prime}(z) D(z)=D(p z) C(z),
$$

then $M(z) D(z)^{t}$ is a fundamental matrix for a $q$-difference equation with associated $p$-difference equation $w(p z)=C^{\prime}(z) w(z)$.

Definition 2.10. The weak monodromy of a $p$-theta $q$-difference equation is the associated equivalence class of $q$-theta $p$-difference equations. Two $p$-theta $q$-difference equations are isomonodromic if they have the same weak monodromy.

Theorem 2.11. The p-theta $q$-difference equations $v(q z)=A(z) v(z), v(q z)=A^{\prime}(z) v(z)$ are isomonodromic iff there exists a nonsingular p-elliptic matrix $B(z)$ such that

$$
A^{\prime}(z) B(z)=B(q z) A(z) .
$$

Proof. Choose a $q$-theta $p$-difference equation $w(q z)=C(z) w(z)$ representing the weak monodromy of the first equation. The equations are isomonodromic iff $w(q z)=C(z) w(z)$ represents the weak monodromy of the second equation, iff the two equations have fundamental matrices satisfying $M(q z)=M(z) C(z)$. But by Theorem 2.9 (swapping $p$ and $q$ ), this holds iff $A^{\prime}(z) B(z)=B(q z) A(z)$ for some $p$-elliptic matrix $B(z)$. 
Remark 2.12. Compare [5], where the analogous result is proved for difference equations, relative to Birkhoff's [4] notion of monodromy.

Corollary 2.13. The map from isomonodromy classes of p-theta q-difference equations to their weak monodromies is well-defined, and inverse to the map from isomonodromy classes of q-theta p-difference equations to their weak monodromies.

Remark 2.14. The isomonodromy equivalence relation is also quite natural from the perspective of the general theory of difference equations (see, e.g., [17]); to be precise, two $p$-theta $q$-difference equations are isomonodromic iff they induce isomorphic difference modules. The latter fact induces a natural isomorphism between their difference Galois groups (at least when the latter are defined, i.e., when the equations are elliptic), as can be seen directly from the interpretation of difference Galois groups via Tannakian categories. This preservation of Galois groups seems to be what is truly intended by the word "isomonodromy", even in the differential setting. For instance, for non-Fuchsian equations, where the monodromy group conveys relatively little information, one only obtains the relevant Painlevé equations by insisting that the corresponding deformations should preserve Stokes data as well.

It will be convenient in the sequel to introduce a slightly weaker equivalence relation.

Definition 2.15. Two $p$-theta $q$-difference equations are theta-isomonodromic if there exists a nonsingular meromorphic $p$-theta matrix $B(z)$ such that the shift matrices $A(z), A^{\prime}(z)$ of the equations satisfy $A^{\prime}(z) B(z)=B(q z) A(z)$.

Theorem 2.16. Two p-theta q-difference equations are theta-isomonodromic iff their weak monodromies agree up to multiplication of the shift matrix by a factor of the form a $z^{k}$. $A(z)$.

Note that $v(q z)=A(z) v(z)$ and $v(q z)=A(q z) v(z)$ are theta-isomonodromic with $B(z)=$

Remark 2.17. Though this equivalence relation no longer preserves Galois groups, even if both equations are elliptic, it comes quite close to doing so. Indeed, the Galois group of an $n$-th order equation is naturally a subgroup of $\mathrm{GL}_{n}$, and we may thus consider its image in $\mathrm{PGL}_{n}$, which one might call the projective Galois group. Since $\mathrm{PGL}_{n}$ is the image of $\mathrm{GL}_{n}$ under the adjoint representation, one finds that the projective Galois group of the equation with shift matrix $A(z)$ can be identified with the ordinary Galois group of the equation with shift matrix $A(z) \otimes A(z)^{-t}$. If two equations are theta-isomonodromic, their images under the adjoint representation are thus fully isomonodromic, and thus the original equations had the same projective Galois groups. This even extends to $p$-theta $q$-difference equations once we observe that the image of such an equation under the adjoint representation is elliptic, and thus the projective Galois groups of such equations are still well-defined.

Remark 2.18. The relation of isomonodromy to Galois groups suggests some further questions, which are in the main outside the scope of the current paper, but seem to merit a brief mention nonetheless.

First, since (theta-)isomonodromic equations have isomorphic (projective) Galois groups, it is natural to ask whether one can recover the (projective) Galois group more directly from the weak monodromy. Since the weak monodromy is itself a isomonodromy class, any two representatives of the weak monodromy have the same Galois group, and one would expect that group to be related to the original Galois group. It can be shown (Etingof, personal communication) that in fact the groups are naturally isomorphic, with dual associated representations. Thus, for instance, the fact that the difference equations we will be considering have triangular weak 
monodromy implies that they have solvable Galois group. (This also follows immediately from the fact that, by construction, they have theta function solutions.)

Another natural question is whether there exists a stronger notion of monodromy; for rational $q$-difference equations with sufficiently nice singularities, there is a well-defined notion of monodromy, an associated nonsingular $q$-elliptic matrix the nonsingular values of which generate a Zariski dense subgroup of the Galois group ([9]; see also Chapter 12 of [17]). Krichever [14] defines an analogous matrix for generic difference equations with theta function coefficients (although the relation to the Galois group is again unclear); although Krichever's genericity assumptions explicitly exclude the situation we consider above (raising the question of whether there is an analogue in our setting), his monodromy is again a difference equation with theta function coefficients. This suggests that the rational $q$-difference notion of monodromy should correspond at the elliptic level to a representative of our weak monodromy, and thus suggests the question of whether given a $p$-elliptic $q$-difference equation, there exists a representative of its weak monodromy such that the nonsingular values of the corresponding $C$ matrix are Zariski dense in its Galois group.

\section{Semiclassical biorthogonal elliptic functions}

In [22], Spiridonov constructed a family of elliptic hypergeometric functions biorthogonal with respect to the density of the elliptic beta integral:

$$
\frac{(p ; p)(q ; q)}{2} \int_{C} \frac{\prod_{0 \leq r<6} \Gamma_{p, q}\left(u_{r} z^{ \pm 1}\right)}{\Gamma_{p, q}\left(z^{ \pm 2}\right)} \frac{d z}{2 \pi \sqrt{-1} z}=\prod_{0 \leq r<s<6} \Gamma_{p, q}\left(u_{r} u_{s}\right),
$$

where the parameters satisfy the balancing condition

$$
\prod_{0 \leq r<6} u_{r}=p q
$$

and the (possibly disconnected, but closed) contour is chosen to be symmetrical under $z \mapsto 1 / z$, and to contain all points of the form $p^{i} q^{j} u_{r}, i, j \geq 0,0 \leq r<6$, or more precisely, all poles of the integrand of that form.

If we view this as the "classical" case, then this suggests, by analogy with $[15,11]$ that we should study biorthogonal functions with respect to the more general density

$$
\Delta^{(m)}\left(z ; u_{0}, \ldots, u_{2 m+5}\right)=\frac{\prod_{0 \leq r<2 m+6} \Gamma_{p, q}\left(u_{r} z^{ \pm 1}\right)}{\Gamma_{p, q}\left(z^{ \pm 2}\right)},
$$

with new balancing condition

$$
\prod_{0 \leq r<2 m+6} u_{r}=(p q)^{m+1},
$$

and the corresponding contour condition, integrated against the differential

$$
\frac{(p ; p)(q ; q)}{2} \frac{d z}{2 \pi \sqrt{-1} z} \text {. }
$$

Note that if $u_{2 m+4} u_{2 m+5}=p q$, then the corresponding factors of the density cancel, and thus we reduce to the order $m-1$ density. Also, it will be convenient to multiply the integrands by theta functions, not elliptic functions; such multiplication has the effect of shifting the balancing condition. (The extent of the required shift can be determined via the observation that 
multiplying a parameter by $q$ multiplies the integrand by a $p$-theta function; in any event, we will give the explicit balancing condition for each of the integrals appearing below.)

One natural multivariate analogue of the elliptic beta integral is the elliptic Selberg integral $[7,19]$, the higher-order version of which we define as follows

$$
\begin{aligned}
& I I_{n ; t ; p, q}^{(m)}\left(u_{0}, \ldots, u_{2 m+5}\right) \\
& \quad:=\frac{(p, p)^{n}(q ; q)^{n}}{\Gamma_{p, q}(t)^{-n} 2^{n} n !} \int_{C^{n}} \prod_{1 \leq i<j \leq n} \frac{\Gamma_{p, q}\left(t z_{i}^{ \pm 1} z_{j}^{ \pm 1}\right)}{\Gamma_{p, q}\left(z_{i}^{ \pm 1} z_{j}^{ \pm 1}\right)} \prod_{1 \leq i \leq n} \frac{\prod_{0 \leq r<2 m+6} \Gamma_{p, q}\left(u_{r} z_{i}^{ \pm 1}\right)}{\Gamma_{p, q}\left(z_{i}^{ \pm 2}\right)} \frac{d z_{i}}{2 \pi \sqrt{-1} z_{i}},
\end{aligned}
$$

where the parameters satisfy the conditions $|t|,|p|,|q|<1$, and

$$
t^{2 n-2} \prod_{0 \leq r<2 m+6} u_{r}=(p q)^{m+1}
$$

and the contour $C$ is chosen so that $C=C^{-1}$, and such that the interior of $C$ contains every contour of the form $p^{i} q^{j} t C, i, j \geq 0$, and every point of the form $p^{i} q^{j} u_{r}, i, j \geq 0,0 \leq r<2 m+6$. (The latter set of points represents poles of the integrand; if (as often occurs below) some of these points are not poles, then the corresponding contour condition can of course be removed. Similarly, if the cross terms are holomorphic (e.g., if $t=q$, as is the case below), then $C$ need not contain the contours $p^{i} q^{j} t C$.) Note that if $\left|u_{0}\right|, \ldots,\left|u_{2 m+5}\right|<1$, then $C$ can be chosen to be the unit circle. More generally, such a contour exists as long as $p^{i} q^{j} t^{k} u_{r} u_{s}$ is never 1 for $i, j, k \geq 0$, $0 \leq r, s<2 m+6$, and the result is a meromorphic function on the parameter domain.

When $m=0$, the elliptic Selberg integral can be explicitly evaluated [19, Theorem 6.1]:

$$
I I_{n ; t ; p, q}^{(0)}\left(u_{0}, u_{1}, u_{2}, u_{3}, u_{4}, u_{5}\right)=\prod_{0 \leq i<n} \Gamma_{p, q}\left(t^{i+1}\right) \prod_{0 \leq r<s<6} \Gamma_{p, q}\left(t^{i} u_{r} u_{s}\right)
$$

while the order 1 elliptic Selberg integral satisfies a transformation law with respect to the Weyl group $E_{7}$; more precisely, the renormalized (holomorphic) function

$$
\tilde{I}_{n ; t ; p, q}^{(1)}\left(u_{0}, \ldots, u_{7}\right):=I I_{n ; t ; p, q}^{(1)}\left(t^{1 / 2} u_{0}, \ldots, t^{1 / 2} u_{7}\right) \prod_{0 \leq r<s<8} \Gamma_{p, q, t}^{+}\left(t u_{r} u_{s}\right)
$$

is invariant under the natural action of $E_{7}$ on the torus of parameters [19, Corollary 9.11]. More importantly for our present purposes, when $t=q$, the renormalized order 1 elliptic Selberg integral satisfies an $E_{8}$-invariant family of nonlinear difference equations making it a tau function for the elliptic Painlevé equation [18, Theorem 5.1] (for the relevant definition of tau functions, see [12]). As an aside, it should be noted that [18] also showed that when $t=q^{1 / 2}$ or $t=q^{2}$, the integral satisfies slightly more complicated analogues of the tau function identities; as yet, neither a geometric nor an isomonodromy interpretation of those identities is known.

Since we will be fixing $p, q$, and $t=q$ in the sequel, we omit these parameters from the notation; we will also generally omit $m$, as it can be determined by counting the arguments.

Consider the following instance of the elliptic Selberg integral:

$$
F_{n}(x ; v)=x^{-n} v^{n} I I_{n}\left(u_{0}, \ldots, u_{2 m+5}, q x, p q / x, v, p / v\right),
$$

satisfying, as usual, the balancing condition

$$
q^{2 n-2} \prod_{0 \leq r<2 m+6} u_{r}=(p q)^{m+1} .
$$


Since

$$
\psi_{p}(x, z):=x^{-1} \Gamma_{p, q}\left(q x z^{ \pm 1}, p q / x z^{ \pm 1}\right)=\frac{\Gamma_{p, q}\left(q x z^{ \pm 1}\right)}{x \Gamma_{p, q}\left(x z^{ \pm 1}\right)}=x^{-1} \theta_{p}\left(x z^{ \pm 1}\right),
$$

we see that the integrand of $F_{n}(x ; v)$ is holomorphic in $x$; indeed, it differs from the order $m$ elliptic Selberg integrand by a factor

$$
\prod_{1 \leq i \leq n} \frac{\psi_{p}\left(x, z_{i}\right)}{\psi_{p}\left(v, z_{i}\right)}
$$

In particular, the $x$-dependent conditions on the contour are irrelevant, as there are no $x$ dependent poles. We thus find that $F_{n}(x ; v)$ is a $B C_{1}$-symmetric theta function of degree $n$; that is, it is a holomorphic function of $x$ satisfying

$$
F_{n}(1 / x ; v)=F_{n}(x ; v), \quad F_{n}(p x ; v)=\left(p x^{2}\right)^{-n} F_{n}(x ; v) .
$$

(In general, $B C_{n}$ denotes the "hyperoctahedral" group of signed permutations, which will act by permutations and taking reciprocals.) This function satisfies a form of biorthogonality; to be precise, we have the following.

Theorem 3.1. Let $G_{n}(x)$ be any $B C_{1}$-symmetric theta function of degree $n$, and let $C$ be any contour satisfying the constraints corresponding to the parameters $u_{0}, \ldots, u_{2 m+5}, v, p / v$ with

$$
q^{2 n-2} \prod_{0 \leq r<2 m+6} u_{r}=(p q)^{m+1} .
$$

Then for any $x$ such that the contour $C$ contains $p^{i} x$ and $p^{i+1} / x$ for all $i \geq 0$,

$$
\begin{aligned}
& \frac{(p ; p)^{2}}{2} \int_{C} \frac{F_{n}(z ; v) G_{n}(z)}{\psi_{p}(x, z) \psi_{p}(v, z)} \Delta\left(z ; u_{0}, \ldots, u_{2 m+5}\right) \frac{d z}{2 \pi \sqrt{-1} z} \\
& \quad=G_{n}(x) x^{n+1} v^{n+1} I I_{n+1}\left(u_{0}, \ldots, u_{2 m+5}, x, p / x, v, p / v\right) .
\end{aligned}
$$

In particular, if $H_{n-1}(x)$ is a $B C_{1}$-symmetric theta function of degree $n-1$, then

$$
\int_{C} \frac{F_{n}(z ; v) H_{n-1}(z)}{\psi_{p}(v, z)} \Delta\left(z ; u_{0}, \ldots, u_{2 m+5}\right) \frac{d z}{2 \pi \sqrt{-1} z}=0 .
$$

Proof. If replace $F_{n}(z ; v)$ by its definition, the result is an $n+1$-dimensional contour integral over $C^{n+1}$. Moreover, the integrand is very nearly symmetric between $z$ and the remaining $n$ integration variables. To be precise, if we write the original integration variable as $z_{0}$, then the resulting integrand is a $B C_{n+1}$-symmetric factor multiplied by

$$
\frac{G_{n}\left(z_{0}\right)}{\psi_{p}\left(x, z_{0}\right) \prod_{1 \leq i \leq n} \psi_{p}\left(z_{0}, z_{i}\right)},
$$

which is invariant under the subgroup $B C_{1} \times B C_{n}$. If we average the integrand over $B C_{n+1}$, this will not change the integral, as the contour is $B C_{n+1}$-invariant. We can thus replace the above factor by the average over cosets:

$$
\frac{1}{n+1} \sum_{0 \leq k \leq n} \frac{G_{n}\left(z_{k}\right)}{\psi_{p}\left(x, z_{k}\right) \prod_{i \neq k} \psi_{p}\left(z_{k}, z_{i}\right)}=\frac{1}{n+1} \frac{G_{n}(x)}{\prod_{0 \leq i \leq n} \psi_{p}\left(x, z_{i}\right)} ;
$$

the identity follows from the fact that if we multiply both sides by $\prod_{0 \leq i \leq n} \psi_{p}\left(x, z_{i}\right)$, then both sides are $B C_{1}$-symmetric theta functions of degree $n$ in $x$, and agree at the $n+1$ distinct pairs of points $z_{i}^{ \pm 1}$.

The claim follows immediately. 
Remark 3.2. At the level of orthogonal polynomials, such an $n$-dimensional integral representation is implicit in [25]; more precisely, Szegö gives a representation of orthogonal polynomials as a determinant, but the Cauchy-Binet identity allows one to turn it into an $n$-dimensional integral involving the square of a Vandermonde determinant.

Note that in the above calculation, the $x$-dependent constraint on the contour was only relevant to the eventual identification of the $n+1$-dimensional integral as an elliptic Selberg integral. We also observe that if $v$ has the form $u_{r} / q$, then the parameters $u_{r}$ and $p / v$ in the Selberg integrals multiply to $p q$ and thus cancel. We thus find that $F_{n}\left(z ; u_{r} / q\right)$ satisfies biorthogonality with respect to a general order $m$ instance of $\Delta(z)$. It will, however, be convenient to allow general $v$ in the sequel.

We thus see that the integral $F_{n}(z ; v)$ is in some sense an analogue of an orthogonal polynomial. Similarly, the $n+1$-dimensional integral of Theorem 3.1 is analogous to a Cauchy transform of $F_{n}(z ; v)$, as the integral of $F_{n}(z ; v)$ against a function with a moving pole. This suggests that these two integrals should form a row in the fundamental matrix of our difference equation. This leads to the question of how this row depends on $v$. Define

$$
F_{n}^{+}(x, v)=v^{n+1} x^{n+1} \psi_{p}(v, x) I I_{n+1}\left(u_{0}, \ldots, u_{2 m+5}, x, p / x, v, p / v\right),
$$

where the factor $x^{n+1}$ is chosen to make the integrand invariant under $x \mapsto 1 / x$, the factor $v^{n+1}$ for symmetry, and the factor $\psi_{p}(v, x)$ to simplify the following identity.

Lemma 3.3. The functions $F_{n}(x ; v)$ and $F_{n}^{+}(x ; v)$ satisfy the identity

$$
F_{n}(x ; v) F_{n}^{+}(x, w)-F_{n}(x ; w) F_{n}^{+}(x, v)=I I_{n}\left(u_{0}, \ldots, u_{2 m+5}\right) F_{n}^{+}(v, w) .
$$

Proof. Taking $G_{n}(x)=F_{n}(x ; w)$ in Theorem 3.1 gives

$$
\begin{aligned}
& F_{n}(x ; w) F_{n}^{+}(x, v) \\
& \quad=\frac{(p ; p)^{2}}{2} \int_{C} \frac{\psi_{p}(v, x)}{\psi_{p}(x, z) \psi_{p}(v, z)} F_{n}(z ; v) F_{n}(z ; w) \Delta\left(z ; u_{0}, \ldots, u_{2 m+5}\right) \frac{d z}{2 \pi \sqrt{-1} z} .
\end{aligned}
$$

Thus the two terms on the left-hand side agree except in the first factors of the integrands; the difference of the two integrals can be simplified using the addition law, and gives a result independent of $x$; setting $x=v$ gives the desired result.

Similarly, we have the following. Let

$$
F_{n}^{-}(x, v):=\psi_{p}(v, x) x^{1-n} v^{1-n} I I_{n-1}\left(u_{0}, \ldots, u_{2 m+5}, q x, p q / x, q v, p q / v\right) .
$$

Lemma 3.4. For any $B C_{1}$-symmetric theta function $G_{n}$ of degree $n$,

$$
\begin{aligned}
& \frac{(p ; p)^{2}}{2} \int_{C} \frac{F_{n}^{-}(z, v) G_{n}(z) \psi_{p}(x, y)}{\psi_{p}(x, z) \psi_{p}(y, z)} \Delta\left(z ; u_{0}, \ldots, u_{2 m+5}\right) \frac{d z}{2 \pi \sqrt{-1} z} \\
& \quad=G_{n}(x) F_{n}(v ; x)-G_{n}(y) F_{n}(v ; y) .
\end{aligned}
$$

In particular,

$$
F_{n}^{-}(x, v) F_{n}^{+}(x, w)+F_{n}(v ; x) F_{n}(x ; w)=I I_{n}\left(u_{0}, \ldots, u_{2 m+5}\right) F_{n}(v ; w),
$$

and if $H_{n-2}(z)$ is any $B C_{1}$-symmetric theta function of degree $n-2$,

$$
\int_{C} F_{n}^{-}(z, v) H_{n-2}(z) \Delta\left(z ; u_{0}, \ldots, u_{2 m+5}\right) \frac{d z}{2 \pi \sqrt{-1} z}=0 .
$$


Remark 3.5. In particular, we see that

$$
\frac{F_{n}^{-}(z, v)}{\psi_{p}(v, z)}
$$

is essentially a biorthogonal function of degree $n-1$.

Theorem 3.6. The functions $F_{n}^{-}, F_{n}$ and $F_{n}^{+}$satisfy the identities

$$
\begin{aligned}
& F_{n}^{+}(v, w)\left(\begin{array}{c}
F_{n}(x ; u) \\
F_{n}^{+}(x, u)
\end{array}\right)-F_{n}^{+}(u, w)\left(\begin{array}{c}
F_{n}(x ; v) \\
F_{n}^{+}(x, v)
\end{array}\right)+F_{n}^{+}(u, v)\left(\begin{array}{c}
F_{n}(x ; w) \\
F_{n}^{+}(x, w)
\end{array}\right)=0, \\
& F_{n}^{+}(v, w)\left(\begin{array}{c}
F_{n}^{-}(x, u) \\
-F_{n}(u ; x)
\end{array}\right)-F_{n}(u ; w)\left(\begin{array}{c}
F_{n}(x ; v) \\
F_{n}^{+}(x, v)
\end{array}\right)+F_{n}(u ; v)\left(\begin{array}{c}
F_{n}(x ; w) \\
F_{n}^{+}(x, w)
\end{array}\right)=0, \\
& F_{n}(v ; w)\left(\begin{array}{c}
F_{n}^{-}(x, u) \\
-F_{n}(u ; x)
\end{array}\right)-F_{n}(u ; w)\left(\begin{array}{c}
F_{n}^{-}(x, v) \\
-F_{n}(v ; x)
\end{array}\right)+F_{n}^{-}(u, v)\left(\begin{array}{c}
F_{n}(x ; w) \\
F_{n}^{+}(x, w)
\end{array}\right)=0, \\
& F_{n}^{-}(v, w)\left(\begin{array}{c}
F_{n}^{-}(x, u) \\
-F_{n}(u ; x)
\end{array}\right)-F_{n}^{-}(u, w)\left(\begin{array}{c}
F_{n}^{-}(x, v) \\
-F_{n}(v ; x)
\end{array}\right)+F_{n}^{-}(u, v)\left(\begin{array}{c}
F_{n}^{-}(x, w) \\
-F_{n}(w ; x)
\end{array}\right)=0 .
\end{aligned}
$$

Proof. Each identity is the Plücker relation for the $2 \times 3$ matrix formed by concatenating the three column vectors that appear. In the first two cases, we have already computed the requisite minors; the remaining minor follows as a special case of the third identity, which can be derived by eliminating a common term from two instances of the second identity.

Remark 3.7. Note that the proof of these identities didn't require the balancing condition, or even that the biorthogonality density was $\Delta$. Furthermore, the only way in which the proof depended on properties of elliptic functions was in the fact that $\psi_{p}$ satisfies a partial fraction decomposition result. If we generalize the results with this in mind, we find that these are precisely the generalized Fay identities of $[1,18]$.

We also note that the change of basis from $F_{n}$ to $F_{n}^{-}$can be interpreted as relating degree $n$ biorthogonal functions to degree $n-1$ biorthogonal functions; i.e., the analogue of the three-term recurrence for orthogonal polynomials.

We thus see that, as functions of $x$, the vectors

$$
\left(\begin{array}{c}
F_{n}(x ; v) \\
F_{n}^{+}(x, v)
\end{array}\right)
$$

and

$$
\left(\begin{array}{c}
F_{n}^{-}(x, v) \\
-F_{n}(v ; x)
\end{array}\right)
$$

for all $v \in \mathbb{C}^{*}$, together span only a 2-dimensional space, and the change of basis matrix between any two such bases of this 2-dimensional space is computable in terms of $F_{n}, F_{n}^{ \pm}$. And, naturally, the choice of basis will have no effect on the resulting difference equation beyond conjugation by a matrix independent of $x$. Since it will be useful to allow fairly general bases, we extend the notation by defining values for $F_{n}^{+}$on hatted arguments (equivalently, defining $F_{n}^{+}$as a function on $\left(\mathbb{C}^{*} \uplus \mathbb{C}^{*}\right)^{2}$, where $\mathbb{C}^{*} \uplus \mathbb{C}^{*}$ is the disjoint union of two copies of $\mathbb{C}^{*}$; thus a given element $v \in \mathbb{C}^{*}$ corresponds to two elements of $\mathbb{C}^{*} \uplus \mathbb{C}^{*}$, denoted by $v$ and $\hat{v}$ respectively), as follows:

$$
\begin{array}{ll}
F_{n}^{+}(\hat{v}, w):=F_{n}(v ; w), & F_{n}^{+}(v, \hat{w}):=-F_{n}(w ; v), \\
F_{n}^{+}(\hat{v}, \hat{w}):=F_{n}^{-}(v, w), & F_{n}(x ; \hat{v}):=F_{n}^{-}(x, v)
\end{array}
$$


note that this extension of $F_{n}^{+}$preserves its antisymmetry. Note that in this notation, the identities relating $F_{n}, F_{n}^{ \pm}$reduce to the single identity

$$
F_{n}^{+}(w, x) F_{n}^{+}(y, z)-F_{n}^{+}(w, y) F_{n}^{+}(x, z)+F_{n}^{+}(w, z) F_{n}^{+}(x, y)=0,
$$

for all $w, x, y, z \in \mathbb{C}^{*} \uplus \mathbb{C}^{*}$, and the minors used in the Plücker relations follow from the special case

$$
F_{n}^{+}(\hat{v}, v)=F_{n}(v ; v)=I I_{n}\left(u_{0}, \ldots, u_{2 m+5}\right) .
$$

To proceed further, we will need to understand how our functions behave under the monodromy action $x \mapsto p x$; it will also turn out to be useful to know how $x \mapsto 1 / x$ acts. Easiest of all is $x \mapsto p / x$; in that case, the elliptic Selberg integral itself is manifestly invariant, so we need simply consider how the prefactors transform:

$$
F_{n}(p / x ; v)=\left(x^{2} / p\right)^{n} F_{n}(x ; v), \quad F_{n}^{+}(p / x, v)=\left(p / x^{2}\right)^{n} F_{n}^{+}(x, v) .
$$

For $x \mapsto 1 / x$, we similarly have

$$
F_{n}(1 / x ; v)=F_{n}(x ; v) .
$$

However, for $F_{n}^{+}(1 / x, v)$, while the integrand remains constant, the constraints on the contour change. Assume for the moment that $v \in \mathbb{C}^{*}$, and choose a $B C_{1}$-symmetric theta function $G_{n}(z)$ of degree $n$ such that $G_{n}(x) \neq 0$, so that

$$
F_{n}^{+}(x, v)=\frac{(p ; p)^{2}}{2} \int_{C} \frac{\psi_{p}(v, x) F_{n}(z ; v) G_{n}(z)}{\psi_{p}(x, z) \psi_{p}(v, z) G_{n}(x)} \Delta\left(z ; u_{0}, \ldots, u_{2 m+5}\right) \frac{d z}{2 \pi \sqrt{-1} z} .
$$

Then $x \mapsto 1 / x$ leaves the integrand the same, but moves the contour through $x$ and $1 / x$. Thus $F_{n}^{+}(1 / x, v)-F_{n}^{+}(x, v)$ can be computed by residue calculus; by symmetry, we find that it is twice the residue at $z=1 / x$ :

$$
F_{n}^{+}(1 / x, v)-F_{n}^{+}(x, v)=x^{-1} \theta_{q}\left(x^{2}\right) F_{n}(x ; v) \prod_{0 \leq r<2 m+6} \Gamma_{p, q}\left(u_{r} x^{ \pm 1}\right) .
$$

Putting this together, we obtain the following.

Lemma 3.8. The functions $F_{n}$ and $F_{n}^{+}$have the monodromy action

$$
\left(F_{n}(1 / x ; v) \quad F_{n}^{+}(1 / x, v)\right)=\left(F_{n}(x ; v) \quad F_{n}^{+}(x, v)\right)\left(\begin{array}{cc}
1 & x^{-1} \theta_{q}\left(x^{2}\right) \\
0 & \prod_{0 \leq r<2 m+6} \Gamma_{p, q}\left(u_{r} x^{ \pm 1}\right) \\
0 & 1
\end{array}\right)
$$

and

$$
\begin{aligned}
& \left(\begin{array}{ll}
F_{n}(p x ; v) & F_{n}^{+}(p x, v)
\end{array}\right) \\
& \quad=\left(\begin{array}{ll}
F_{n}(x ; v) & F_{n}^{+}(x, v)
\end{array}\right)\left(\begin{array}{cc}
\left(p x^{2}\right)^{-n} & \left(p x^{2}\right)^{n} x^{-1} \theta_{q}\left(x^{2}\right) \\
0 & \prod_{0 \leq r<2 m+6} \Gamma_{p, q}\left(u_{r} x^{ \pm 1}\right) \\
0 & \left(p x^{2}\right)^{n}
\end{array}\right)
\end{aligned}
$$

valid for all $v \in \mathbb{C}^{*} \uplus \mathbb{C}^{*}$.

Proof. The only thing to check is that it extends to the other copy of $\mathbb{C}^{*}$, but this follows immediately from the facts that the monodromy is independent of $v \in \mathbb{C}^{*}$, and that for all $v \in \mathbb{C}^{*} \uplus \mathbb{C}^{*}$, the row vectors lie in the same 2 -dimensional space. 
This is not quite a $q$-theta $p$-difference equation as we would wish, but it is straightforward to turn it into a $q$-theta $p$-difference equation. Define a $2 \times 2$ meromorphic matrix $M_{n}(z ; v, w)$ for $v, w \in \mathbb{C}^{*} \uplus \mathbb{C}^{*}$ :

$$
M_{n}(z ; v, w):=\left(\begin{array}{cc}
F_{n}(z ; v) & z^{-1} \theta_{p}\left(z^{2}\right) F_{n}^{+}(z, v) / \Delta\left(z ; u_{0}, \ldots, u_{2 m+5}\right) \\
F_{n}(z ; w) & z^{-1} \theta_{p}\left(z^{2}\right) F_{n}^{+}(z, w) / \Delta\left(z ; u_{0}, \ldots, u_{2 m+5}\right)
\end{array}\right) .
$$

Theorem 3.9. The matrix $M_{n}(z ; v, w)$ is a meromorphic fundamental matrix for a p-theta $q$ difference equation with multiplier $q^{-2 n}$. The isomonodromy class of the equation is independent of $v$ and $w$, and invariant under all permutations of the parameters and all shifts

$$
\left(u_{0}, \ldots, u_{2 m+5}\right) \mapsto\left(q^{k_{0}} u_{0}, \ldots, q^{k_{2 m+5}} u_{2 m+5}\right)
$$

with $k_{r} \in \mathbb{Z}$ such that

$$
\sum_{0 \leq r<2 m+6} k_{r}=0
$$

and invariant under simultaneous negation of all parameters. The theta-isomonodromy class is further invariant under all shifts

$$
\left(u_{0}, \ldots, u_{2 m+5}, z, n\right) \mapsto\left(q^{k_{0}} u_{0}, \ldots, q^{k_{2 m+5}} u_{2 m+5}, q^{l} z, n+\nu\right)
$$

with $l \in \frac{1}{2} \mathbb{Z}, k_{r} \in l+\mathbb{Z}, \nu \in \mathbb{Z}$ such that

$$
2 \nu+\sum_{0 \leq r<2 m+6} k_{r}=0 .
$$

In addition, the associated shift matrix $A(z)$ satisfies the symmetry $A(1 / q z) A(z)=1$.

Proof. Most of the claims follow immediately from the fact that $M(z ; v, w)$ satisfies the $q$-theta $p$-difference equation

$$
\begin{aligned}
& M_{n}(p z ; v, w) \\
& \quad=M_{n}(z ; v, w)\left(\begin{array}{cc}
\left(p z^{2}\right)^{-n} & \left(p z^{2}\right)^{n-2} \Delta\left(z ; u_{0}, \ldots, u_{2 m+5}\right) / \Delta\left(p z ; u_{0}, \ldots, u_{2 m+5}\right) \\
0 & -\left(p z^{2}\right)^{n-2} \Delta\left(z ; u_{0}, \ldots, u_{2 m+5}\right) / \Delta\left(p z ; u_{0}, \ldots, u_{2 m+5}\right)
\end{array}\right),
\end{aligned}
$$

and has determinant

$$
\operatorname{det}\left(M_{n}(z ; v, w)\right)=I I_{n}\left(u_{0}, \ldots, u_{2 m+5}\right) F_{n}^{+}(v, w) \frac{z^{-1} \theta_{p}\left(z^{2}\right)}{\Delta\left(z ; u_{0}, \ldots, u_{2 m+5}\right)},
$$

so is nonsingular. The only additional thing to check for the isomonodromy claims is that

$$
\left(p z^{2}\right)^{2 n-2} \Delta\left(z ; u_{0}, \ldots, u_{2 m+5}\right) / \Delta\left(p z ; u_{0}, \ldots, u_{2 m+5}\right)
$$

is invariant under all of the stated transformations. The symmetry of $A(z)$ follows immediately from the symmetry

$$
M(1 / z ; v, w)=M(z ; v, w)\left(\begin{array}{cc}
1 & 1 \\
0 & -1
\end{array}\right)
$$


Remark 3.10. One can avoid the appearance of theta-isomonodromy above at the cost of introducing some "apparent" singularities, and an additional parameter controlling the location of those singularities. Indeed, if one defines

$$
M_{n}^{\prime}(z ; x ; v, w):=\frac{\Gamma_{p, q}\left(x z^{ \pm 1}\right)}{\Gamma_{p, q}\left(q^{n} x z^{ \pm 1}\right)} M_{n}(z ; v, w),
$$

then $M_{n}^{\prime}$ satisfies the same transformation law as $M$ with respect to $z \mapsto 1 / z$, while under $z \mapsto p z$, one has

$$
\begin{aligned}
& M_{n}^{\prime}(p z ; x ; v, w) \\
& \quad=M_{n}^{\prime}(z ; x ; v, w)\left(\begin{array}{cc}
1 & \left(p z^{2}\right)^{2 n-2} \Delta\left(z ; u_{0}, \ldots, u_{2 m+5}\right) / \Delta\left(p z ; u_{0}, \ldots, u_{2 m+5}\right) \\
0 & -\left(p z^{2}\right)^{2 n-2} \Delta\left(z ; u_{0}, \ldots, u_{2 m+5}\right) / \Delta\left(p z ; u_{0}, \ldots, u_{2 m+5}\right)
\end{array}\right) .
\end{aligned}
$$

Thus the associated shift matrix

$$
A_{n}^{\prime}(z ; x ; v, w)=\frac{\theta_{p}\left(x z, q^{n-1} x / z\right)}{\theta_{p}\left(q^{n} x z, x / q z\right)} A_{n}(z ; v, w)
$$

is elliptic, with the same symmetry as $A$, and every shift

$$
\left(u_{0}, \ldots, u_{2 m+5}, z, n, x\right) \mapsto\left(q^{k_{0}} u_{0}, \ldots, q^{k_{2 m+5}} u_{2 m+5}, q^{l} z, n+\nu, q^{l^{\prime}} x\right)
$$

with $l \in \frac{1}{2} \mathbb{Z}, l^{\prime}, k_{r} \in l+\mathbb{Z}, \nu \in \mathbb{Z}$ such that

$$
2 \nu+\sum_{0 \leq r<2 m+6} k_{r}=0
$$

gives rise to a true isomonodromy transformation of this elliptic difference equation, with associated operator

$$
B^{\prime}(z ; x ; v, w)=\frac{\Gamma_{p, q}\left(q^{l^{\prime}+l} x z, q^{l^{\prime}-l} x / z\right)}{\Gamma_{p, q}(x z, x / z)} \frac{\Gamma_{p, q}\left(q^{n} x z, q^{n} x / z\right)}{\Gamma_{p, q}\left(q^{n+\nu+l+l^{\prime}} x z, q^{n+\nu+l^{\prime}-l} x / z\right)} B(z ; v, w) .
$$

In particular, the isomonodromy transformations differ from the corresponding theta-isomonodromy transformations by a meromorphic theta function factor depending only on $\nu, l^{\prime}, l$. There is also an isomonodromy transformation between $A_{n}^{\prime}(z ; x ; v, w)$ and $A_{n}^{\prime}\left(z ; x^{\prime} ; v, w\right)$, for arbitrary $x^{\prime}$, but the corresponding $B$ matrix is (generically) multiplication by an elliptic function of degree $2 n$. It follows that only those parameter shifts satisfying the integrality condition above can extend to arbitrary solutions of the elliptic Painlevé equation (for which one effectively has noninteger $n$ ).

Remark 3.11. The lattice of possible $k_{r}$ vectors is the lattice $D_{2 m+6}^{+}$obtained by adjoining the vector

$$
(1 / 2,1 / 2, \ldots, 1 / 2,-1 / 2,-1 / 2, \ldots,-1 / 2)
$$

of sum 0 to the root lattice $D_{2 m+6}$. In particular, when $m=1$, this lattice is precisely the root lattice $E_{8}$.

Remark 3.12. The symmetry of $A(z)$ is precisely the condition for the pair of equations

$$
v(q z)=A(z) v(z), \quad v(1 / z)=v(z)
$$


to be formally consistent. It then follows from a different special case of [16, Theorem 3] that there exists a nonsingular meromorphic matrix $\hat{M}$ such that

$$
\hat{M}(q z)=A(z) \hat{M}(z), \quad \hat{M}(1 / z)=\hat{M}(z) .
$$

We can give such a matrix explicitly, for instance

$$
\hat{M}(z)=M(z)\left(\begin{array}{cc}
1 & -1 / 2 \\
0 & 1
\end{array}\right)\left(\begin{array}{cc}
1 & 0 \\
0 & \frac{\theta_{p}\left(a z^{ \pm 1}, b z^{ \pm 1}\right)}{z^{-1} \theta_{p}\left(z^{2}\right)}
\end{array}\right) .
$$

\section{The difference equation}

Naturally, simply knowing the existence of a difference equation with associated isomonodromy transformations is of strictly limited usefulness, so we would like to be more explicit about the equation, and at the very least generators of the group of monodromy-preserving transformations.

The first thing we will need to understand about the shift matrix is the locations of its singularities; i.e., the points where the coefficients have poles or the determinant has a zero. This in turn depends on determining the polar divisor of $F_{n}^{+}$. Define

$$
(x ; p, q):=\prod_{0 \leq i, j}\left(1-p^{i} q^{j} x\right)
$$

with the usual multiple argument conventions.

Lemma 4.1. The function

$$
\left(\prod_{0 \leq r<2 m+6}\left(u_{r} x, p u_{r} / x ; p, q\right)\right) F_{n}^{+}(x, v)
$$

is holomorphic for $x \in \mathbb{C}^{*}$. If $v \in \mathbb{C}^{*}$, the function vanishes at $x=v, x=p / v$.

Proof. As before, assuming $v \in \mathbb{C}^{*}$, we have

$$
\begin{aligned}
F_{n}^{+}(x, v) & =\frac{(p ; p)^{2}}{2} \int_{C} \frac{\psi_{p}(v, x) F_{n}(z ; v) G_{n}(z)}{\psi_{p}(x, z) \psi_{p}(v, z) G_{n}(x)} \Delta\left(z ; u_{0}, \ldots, u_{2 m+5}\right) \frac{d z}{2 \pi \sqrt{-1} z} \\
& =\frac{(p ; p)^{2}}{2} \int_{C} \frac{x v \psi_{p}(v, x) F_{n}(z ; v) G_{n}(z)}{G_{n}(x)} \Delta\left(z ; u_{0}, \ldots, u_{2 m+5}, x, p / x, v, p / v\right) \frac{d z}{2 \pi \sqrt{-1} z} .
\end{aligned}
$$

where $G_{n}(z)$ is any $B C_{1}$-symmetric $p$-theta function of degree $n$ not vanishing at $x$. Since $F_{n}(z ; v)$ is holomorphic in $z$, we may apply Lemma 10.4 of [19] (note that condition 3 of that lemma reduces in our case to the balancing condition) to conclude that

$$
\left(\prod_{0 \leq r<2 m+6}\left(u_{r} x, u_{r} p / x ; p, q\right)\right)\left(x v, p x / v, p v / x, p^{2} / x v ; p, q\right)(x v)^{-1} \psi_{p}(v, x)^{-1} G_{n}(x) F_{n}^{+}(x, v)
$$

is holomorphic in $x$. (The conclusion concerning the $x$-independent poles is not useful to us, as $F_{n}(z ; v)$ certainly has singularities that depend on the remaining parameters.) This nearly gives us the desired result, except for the factor $G_{n}(x)$, which disappears by the fact that $F_{n}^{+}(x, v)$ is independent of $G_{n}$, and the additional factor

$$
\left(q x v, p q x / v, p q v / x, p^{2} q / x v ; p, q\right) \text {. }
$$


This latter factor can be eliminated, and the result extended to $v \in \mathbb{C}^{*} \uplus \mathbb{C}^{*}$, by expressing $F_{n}^{+}(z, v)$ as a linear combination of $F_{n}^{+}(z, w)$ and $F_{n}^{+}\left(z, w^{\prime}\right)$, which for generic $w$ and $w^{\prime} \in \mathbb{C}^{*}$ are holomorphic at the offending points.

Theorem 4.2. The matrix

$$
\begin{aligned}
\tilde{A}_{n}(z ; v, w) & :=\left(q^{-1} z^{-2} \prod_{0 \leq r<2 m+6} \theta_{p}\left(u_{r} z\right)\right) A_{n}(z ; v, w) \\
& =\left(q^{-1} z^{-2} \prod_{0 \leq r<2 m+6} \theta_{p}\left(u_{r} z\right)\right) M_{n}(q z ; v, w) M_{n}(z ; v, w)^{-1}
\end{aligned}
$$

is holomorphic in $z$, with determinant

$$
\operatorname{det}\left(\tilde{A}_{n}(z ; v, w)\right)=\prod_{0 \leq r<2 m+6} \theta_{p}\left(u_{r} z, u_{r} / q z\right),
$$

satisfies the p-theta transformation law

$$
\tilde{A}_{n}(p z ; v, w)=\left(p q z^{2}\right)^{-m-3} \tilde{A}_{n}(z ; v, w),
$$

and has the symmetry

$$
\tilde{A}_{n}(1 / q z ; v, w)=\left(\begin{array}{cc}
0 & -1 \\
1 & 0
\end{array}\right) \tilde{A}_{n}(z ; v, w)^{t}\left(\begin{array}{cc}
0 & 1 \\
-1 & 0
\end{array}\right) .
$$

Proof. The formula for $\operatorname{det}\left(\tilde{A}_{n}(z ; v, w)\right)$ follows immediately from the formula for the determinant $\operatorname{det}\left(M_{n}(z ; v, w)\right)$. Similarly, the fact that $A_{n}(1 / q z ; v, w) A_{n}(z ; v, w)=1$ becomes

$$
\tilde{A}_{n}(1 / q z ; v, w) \tilde{A}_{n}(z ; v, w)=\operatorname{det} \tilde{A}_{n}(z ; v, w) ;
$$

the symmetry of $\tilde{A}_{n}(z ; v, w)$ follows from the usual formula for the inverse of a $2 \times 2$ matrix:

$$
C^{-1}=\operatorname{det}(C)^{-1}\left(\begin{array}{cc}
C_{22} & -C_{12} \\
-C_{21} & C_{11}
\end{array}\right)
$$

Another use of this formula allows us to explicitly write down the inverse of $M_{n}(z ; v, w)$. This, in turn, allows us to express the entries of $\tilde{A}(z)$ as polynomials in $F_{n}$ and $F_{n}^{+}$with coefficients that are (holomorphic) $p$-theta functions in $z$ :

$$
\begin{aligned}
\tilde{A}_{n}(z ; v, w) & =\left(\begin{array}{cc}
F_{n}(q z ; v) & F_{n}^{+}(q z, v) \\
F_{n}(q z ; w) & F_{n}^{+}(q z, w)
\end{array}\right)\left(\begin{array}{cc}
a(z) & 0 \\
0 & b(z)
\end{array}\right)\left(\begin{array}{cc}
F_{n}^{+}(z, w) & -F_{n}^{+}(z, v) \\
-F_{n}(z ; w) & F_{n}(z ; v)
\end{array}\right) \\
& =\left(\begin{array}{ccc}
F_{n}(q z ; v) & b(z) F_{n}^{+}(q z, v) \\
F_{n}(q z ; w) & b(z) F_{n}^{+}(q z, w)
\end{array}\right)\left(\begin{array}{cc}
a(z) F_{n}^{+}(z, w) & -a(z) F_{n}^{+}(z, v) \\
-F_{n}(z ; w) & F_{n}(z ; v)
\end{array}\right),
\end{aligned}
$$

where

$$
a(z)=\frac{q^{-1} z^{-2} \prod_{0 \leq r<2 m+6} \theta_{p}\left(u_{r} z\right)}{I I_{n}\left(u_{0}, \ldots, u_{2 m+5}\right) F_{n}^{+}(v, w)}, \quad b(z)=\frac{q z^{2} \prod_{0 \leq r<2 m+6} \theta_{p}\left(u_{r} / q z\right)}{I I_{n}\left(u_{0}, \ldots, u_{2 m+5}\right) F_{n}^{+}(v, w)} .
$$

In particular, the only possible poles of $\tilde{A}_{n}$ come from poles of $a(z) F_{n}^{+}(z, v), a(z) F_{n}^{+}(z, w)$, $b(z) F_{n}^{+}(q z, v)$ and $b(z) F_{n}^{+}(q z, w)$, or, equivalently, poles of

$$
a(z) \prod_{0 \leq r<2 m+6}\left(u_{r} z, p u_{r} / z ; p, q\right)^{-1} \sim \prod_{0 \leq r<2 m+6} \frac{\left(p / u_{r} z ; p\right)}{\left(u_{r} q z, p u_{r} / z ; p, q\right)}
$$


(where $\sim$ here and below denotes that the two functions have the same zeros and poles) and

$$
b(z) \prod_{0 \leq r<2 m+6}\left(u_{r} q z, p u_{r} / q z ; p, q\right)^{-1} \sim \prod_{0 \leq r<2 m+6} \frac{\left(q z / u_{r} ; p\right)}{\left(u_{r} q z, p u_{r} / z ; p, q\right)} .
$$

It follows that

$$
\prod_{0 \leq r<2 m+6}\left(u_{r} q z, p u_{r} / z ; p, q\right) \tilde{A}_{n}(z ; v, w)
$$

is holomorphic in $z$. But the entries of $\tilde{A}_{n}(z ; v, w)$ are meromorphic $p$-theta functions, and thus their divisors are periodic in $p$. Since the remaining set of potential poles contains no $p$-periodic subset, there are in fact no surviving poles.

We can also compute the value of $\tilde{A}_{n}(z ; v, w)$ at a number of points.

Theorem 4.3. The matrix $\tilde{A}_{n}(z ; v, w)$ has the special values

$$
\begin{aligned}
& \tilde{A}_{n}\left(u_{s} / q ; v, w\right)=\frac{q u_{s}^{-2} \prod_{0 \leq r<2 m+6} \theta_{p}\left(u_{r} u_{s} / q\right)}{I I_{n}\left(u_{0}, \ldots, u_{2 m+5}\right) F_{n}^{+}(v, w)}\left(\begin{array}{c}
F_{n}\left(u_{s} ; v\right) \\
F_{n}\left(u_{s} ; w\right)
\end{array}\right)\left(F_{n}^{+}\left(u_{s} / q, w\right)-F_{n}^{+}\left(u_{s} / q, v\right)\right), \\
& \tilde{A}_{n}\left(1 / u_{s} ; v, w\right)=\frac{q u_{s}^{-2} \prod_{0 \leq r<2 m+6} \theta_{p}\left(u_{r} u_{s} / q\right)}{I I_{n}\left(u_{0}, \ldots, u_{2 m+5}\right) F_{n}^{+}(v, w)}\left(\begin{array}{l}
F_{n}^{+}\left(u_{s} / q, v\right) \\
F_{n}^{+}\left(u_{s} / q, w\right)
\end{array}\right)\left(-F_{n}\left(u_{s} ; w\right) \quad F_{n}\left(u_{s} ; v\right)\right),
\end{aligned}
$$

for $0 \leq s<2 m+6$. In addition, we have the four values

$$
\begin{aligned}
& \tilde{A}_{n}\left(q^{-1 / 2} ; v, w\right)=\prod_{0 \leq r<2 m+6} \theta_{p}\left(u_{r} q^{-1 / 2}\right)\left(\begin{array}{ll}
1 & 0 \\
0 & 1
\end{array}\right), \\
& \tilde{A}_{n}\left(-q^{-1 / 2} ; v, w\right)=\prod_{0 \leq r<2 m+6} \theta_{p}\left(-u_{r} q^{-1 / 2}\right)\left(\begin{array}{ll}
1 & 0 \\
0 & 1
\end{array}\right), \\
& \tilde{A}_{n}\left((p / q)^{1 / 2} ; v, w\right)=q^{-n} p^{-1} \prod_{0 \leq r<2 m+6} \theta_{p}\left(u_{r}(p / q)^{1 / 2}\right)\left(\begin{array}{cc}
1 & 0 \\
0 & 1
\end{array}\right), \\
& \tilde{A}_{n}\left(-(p / q)^{1 / 2} ; v, w\right)=q^{-n} p^{-1} \prod_{0 \leq r<2 m+6} \theta_{p}\left(-u_{r}(p / q)^{1 / 2}\right)\left(\begin{array}{ll}
1 & 0 \\
0 & 1
\end{array}\right)
\end{aligned}
$$

at the ramification points (fixed points of $z \mapsto 1 / q z \operatorname{modulo}\langle p\rangle$ ).

Proof. We first observe that at $z=u_{s} / q, b(z) F_{n}^{+}(q z, v)$ and $b(z) F_{n}^{+}(q z, w)$ vanish, and thus the formula for $\tilde{A}\left(u_{s} / q\right)$ simplifies as stated. The second set of special values follows similarly from the vanishing of $a(z) F_{n}^{+}(z, v)$ and $a(z) F_{n}^{+}(z, w)$ at $z=p / u_{s}$, together with the $p$-theta law of $\tilde{A}$.

When $z= \pm q^{-1 / 2}$, so that $q z=1 / z$, we find $a(z)=b(z), F_{n}(q z, v)=F_{n}(z, v)$, and $F_{n}^{+}(q z, v)=F_{n}^{+}(z, v)$; the last difference vanishes due to the factor $\theta_{q}\left(z^{2}\right)=0$ in the relevant residue. The expression for $\tilde{A}\left( \pm q^{-1 / 2}\right)$ thus simplifies immediately. Similarly, at $z= \pm \sqrt{p / q}$, we have $q z=p / z$, and again the entries immediately simplify.

Note that the symmetry of $\tilde{A}$ and the elementary values at the ramification points imply that the matrix is already determined by its values at $u_{s} / q$ for any $m+2$ values of $s$ (assuming $u_{s}$ are generic); the above special values are thus highly overdetermined. It is also worth noting that if $v$ and $w$ are of the form $u_{r} / q$ or $\widehat{u_{r}}$ (with different values of $r$ ), then all but two pairs of special values can be expressed entirely in terms of order $m$ elliptic Selberg integrals with shifted 
parameters. This specialization also has the effect of causing the kernel of $\tilde{A}_{n}\left(u_{r} / q\right)$ and image of $\tilde{A}_{n}\left(1 / u_{r}\right)$ (or vice versa, as appropriate) to be coordinate vectors. In particular, it follows that cross-ratios of kernel vectors are themselves ratios of order $m$ elliptic Selberg integrals, so long as the four arguments in question do not contain both $u_{s} / q$ and $1 / u_{s}$ for some $s$. For instance, the cross-ratio of the vectors $\operatorname{ker} \tilde{A}_{n}\left(u_{0} / q\right), \ldots, \operatorname{ker} \tilde{A}_{n}\left(u_{3} / q\right)$ is

$$
\frac{F_{n}^{+}\left(u_{0} / q, u_{2} / q\right) F_{n}^{+}\left(u_{1} / q, u_{3} / q\right)}{F_{n}^{+}\left(u_{0} / q, u_{3} / q\right) F_{n}^{+}\left(u_{1} / q, u_{2} / q\right)} .
$$

We also note that when $n=0, F_{0}(z ; \hat{w})=F_{0}^{-}(z, w)=0$, and thus if $v$ is not "hatted", then $\tilde{A}_{0}(z ; v, \hat{w})$ is well-defined and triangular; note in particular that $F_{0}^{+}(v, \hat{w})=1$. In particular, it follows that for any $n \geq 0, \tilde{A}_{n}(z ; v, w)$ is isomonodromic to a triangular shift operator with at most the same number of singularities.

It remains to consider the isomonodromy transformations. Changing $v$ and $w$ is straightforward, as we have already observed; the precise isomonodromy transformation follows from

$$
M_{n}\left(z ; v^{\prime}, w^{\prime}\right)=\frac{1}{F_{n}^{+}(v, w)}\left(\begin{array}{cc}
F_{n}^{+}\left(v^{\prime}, w\right) & -F_{n}^{+}\left(v^{\prime}, v\right) \\
F_{n}^{+}\left(w^{\prime}, w\right) & -F_{n}^{+}\left(w^{\prime}, v\right)
\end{array}\right) M_{n}(z ; v, w) .
$$

With this in mind, we can feel free to make choices for $v$ and $w$ if this will simplify the expressions for the remaining isomonodromy transformations.

We first consider the case of integer shifts, or in other words shifts under the lattice $D_{2 m+6}$. It thus suffices to consider the two cases $\left(u_{0}, u_{1}, n\right) \mapsto\left(q u_{0}, u_{1} / q, n\right),\left(q u_{0}, q u_{1}, n-1\right)$.

Lemma 4.4. We have the (isomonodromy) transformations

$$
\begin{aligned}
& M_{n}\left(z ; u_{0}, \widehat{u_{1} / q} ; q u_{0}, u_{1} / q, u_{2}, \ldots, u_{2 m+5}\right) \\
& \quad=\left(u_{0} q / u_{1}\right)^{n}\left(\begin{array}{cc}
1 & 0 \\
0 & \frac{\theta_{p}\left(u_{1} z^{ \pm 1} / q\right)}{\theta_{p}\left(u_{0} z^{ \pm 1}\right)}
\end{array}\right) M_{n}\left(z ; u_{1} / q, \widehat{u_{0}} ; u_{0}, \ldots, u_{2 m+5}\right)
\end{aligned}
$$

and

$$
\begin{aligned}
& M_{n-1}\left(z ; u_{0}, u_{1} ; q u_{0}, q u_{1}, u_{2}, \ldots, u_{2 m+5}\right) \\
& \quad=\left(u_{0} u_{1}\right)^{n-1}\left(\begin{array}{cc}
\frac{u_{1}}{\theta_{p}\left(u_{1} z^{ \pm 1}\right)} & 0 \\
0 & \frac{u_{0}}{\theta_{p}\left(u_{0} z^{ \pm 1}\right)}
\end{array}\right) M_{n}\left(z ; \widehat{u_{1}}, \widehat{u_{0}} ; u_{0}, \ldots, u_{2 m+5}\right) .
\end{aligned}
$$

Remark 4.5. In the version with apparent singularities controlled by $x$, the transformations (4.1) and (4.2) remain unchanged, while (4.3) should be multiplied by $\theta_{p}\left(q^{n-1} x z^{ \pm 1}\right)$.

It remains to consider the case of a half-integer shift, say

$$
\begin{aligned}
& \left(u_{0}, \ldots, u_{m+2}, u_{m+3}, \ldots, u_{2 m+5}, z, v, w\right) \\
& \quad \mapsto\left(q^{1 / 2} u_{0}, \ldots, q^{1 / 2} u_{m+2}, q^{-1 / 2} u_{m+3}, \ldots, q^{-1 / 2} u_{2 m+5}, q^{1 / 2} z, q^{1 / 2} v^{\prime}, q^{1 / 2} w^{\prime}\right) .
\end{aligned}
$$

In this case, just as with $A$ itself, we cannot give a closed-form expression, so must be content with a description of the singularities and a sufficiently large set of special values. Let $B_{n}\left(z ; v, w ; v^{\prime}, w^{\prime}\right)$ denote this isomonodromy transformation; that is,

$$
B_{n}\left(z ; v, w ; v^{\prime}, w^{\prime}\right)=M_{n}\left(q^{1 / 2} z ; q^{1 / 2} v^{\prime}, q^{1 / 2} w^{\prime} ; u_{0}^{\prime}, \ldots, u_{2 m+5}^{\prime}\right) M_{n}\left(z ; v, w ; u_{0}, \ldots, u_{2 m+5}\right)^{-1},
$$


where

$$
u_{r}^{\prime}= \begin{cases}q^{1 / 2} u_{r}, & 0 \leq r<m+3 \\ q^{-1 / 2} u_{r}, & m+3 \leq r<2 m+6\end{cases}
$$

Note that

$$
B_{n}\left(1 / q z ; v, w ; v^{\prime}, w^{\prime}\right)^{-1} B_{n}\left(z ; v, w ; v^{\prime}, w^{\prime}\right)=A_{n}(z ; v, w) .
$$

For convenience, we define

$$
G_{n}^{+}(v, w):=F_{n}^{+}\left(q^{1 / 2} v, q^{1 / 2} w ; u_{0}^{\prime}, \ldots, u_{2 m+5}^{\prime}\right),
$$

and similarly for $G_{n}(v ; w)$; this will free us to again omit most of the parameters from the functions $F_{n}$ and $F_{n}^{+}$.

Theorem 4.6. The matrix

$$
\tilde{B}_{n}\left(z ; v, w ; v^{\prime}, w^{\prime}\right):=\left(\left(q^{1 / 2} z\right)^{-1} \prod_{0 \leq r<m+3} \theta_{p}\left(u_{r} z\right)\right) B_{n}\left(z ; v, w ; v^{\prime}, w^{\prime}\right)
$$

is holomorphic in $z$ with p-theta law

$$
\tilde{B}_{n}\left(p z ; v, w ; v^{\prime}, w^{\prime}\right)=\left(q^{-n} p^{-1}(-1)^{m+3} \prod_{0 \leq r<m+3} u_{r}^{-1}\right) z^{-m-3} \tilde{B}_{n}\left(z ; v, w ; v^{\prime}, w^{\prime}\right),
$$

has determinant

$$
-q^{-1 / 2} \frac{I I_{n}\left(u_{0}^{\prime}, \ldots, u_{2 m+5}^{\prime}\right) G_{n}^{+}\left(v^{\prime}, w^{\prime}\right)}{I I_{n}\left(u_{0}, \ldots, u_{2 m+5}\right) F_{n}^{+}(v, w)} \prod_{0 \leq r<m+3} \theta_{p}\left(u_{r} z\right) \prod_{m+3 \leq r<2 m+6} \theta_{p}\left(u_{r} / q z\right),
$$

and has the special values

$$
\begin{aligned}
\tilde{B}_{n}\left(1 / u_{s} ; v, w ; v^{\prime}, w^{\prime}\right) & \\
= & \frac{-u_{s}^{-1} \prod_{m+3 \leq r<2 m+6} \theta_{p}\left(u_{r} u_{s} / q\right)}{I I_{n}\left(u_{0}, \ldots, u_{2 m+5}\right) F_{n}^{+}(v, w)}\left(\begin{array}{c}
G_{n}^{+}\left(u_{s} / q, v^{\prime}\right) \\
G_{n}^{+}\left(u_{s} / q, w^{\prime}\right)
\end{array}\right)\left(-F_{n}\left(u_{s} ; w\right) \quad F_{n}\left(u_{s} ; v\right)\right),
\end{aligned}
$$

for $0 \leq s<m+3$, and

$$
\begin{aligned}
& \tilde{B}_{n}\left(u_{s} / q ; v, w ; v^{\prime}, w^{\prime}\right) \\
& \quad=\frac{\left(q^{-1 / 2} u_{s}\right)^{-1} \prod_{0 \leq r<m+3} \theta_{p}\left(u_{r} u_{s} / q\right)}{I I_{n}\left(u_{0}, \ldots, u_{2 m+5}\right) F_{n}^{+}(v, w)}\left(\begin{array}{c}
G_{n}\left(u_{s} / q ; v^{\prime}\right) \\
G_{n}\left(u_{s} / q ; w^{\prime}\right)
\end{array}\right)\left(F_{n}^{+}\left(u_{s} / q, w\right)-F_{n}^{+}\left(u_{s} / q, v\right)\right)
\end{aligned}
$$

for $m+3 \leq s<2 m+6$.

Proof. As in the computation for $\tilde{A}_{n}$, we can use the known determinant of $M_{n}$ to write the entries of $\tilde{B}_{n}$ in terms of $F_{n}, F_{n}^{+}, G_{n}$, and $G_{n}^{+}$. We find

$$
\tilde{B}_{n}\left(z ; v, w ; v^{\prime}, w^{\prime}\right)=\left(\begin{array}{cc}
G_{n}\left(z ; v^{\prime}\right) & d(z) G_{n}^{+}\left(z, v^{\prime}\right) \\
G_{n}\left(z ; w^{\prime}\right) & d(z) G_{n}^{+}\left(z, w^{\prime}\right)
\end{array}\right)\left(\begin{array}{cc}
c(z) F_{n}^{+}(z, w) & -c(z) F_{n}^{+}(z, v) \\
-F_{n}(z ; w) & F_{n}(z ; v)
\end{array}\right),
$$


where

$$
c(z)=\frac{\left(q^{1 / 2} z\right)^{-1} \prod_{0 \leq r<m+3} \theta_{p}\left(u_{r} z\right)}{I I_{n}\left(u_{0}, \ldots, u_{2 m+5}\right) F_{n}^{+}(v, w)}, \quad d(z)=\frac{-z \prod_{m+3 \leq r<2 m+6} \theta_{p}\left(u_{r} / q z\right)}{I I_{n}\left(u_{0}, \ldots, u_{2 m+5}\right) F_{n}^{+}(v, w)} .
$$

Since

$$
\begin{aligned}
& c(z) F_{n}^{+}(z, v) \sim \frac{\prod_{0 \leq r<m+3}\left(p / u_{r} z ; p\right)}{\prod_{0 \leq r<m+3}\left(q u_{r} z, p u_{r} / z ; p, q\right) \prod_{m+3 \leq r<2 m+6}\left(u_{r} z, p u_{r} / z ; p, q\right)}, \\
& d(z) G_{n}^{+}\left(z, v^{\prime}\right) \sim \frac{\prod_{0 \leq r<m+3}\left(q u_{r} z, p u_{r} / z ; p, q\right) \prod_{m+3 \leq r<2 m+6}\left(q z / u_{r} ; p\right)}{\prod_{m+3 \leq 2 m+6}\left(u_{r} z, p u_{r} / z ; p, q\right)},
\end{aligned}
$$

and the coefficients of $\tilde{B}_{n}$ are $p$-theta functions, we conclude as before that $\tilde{B}_{n}$ is holomorphic.

The special values again follow by choosing $z$ so that $c(z)$ or $d(z)$ vanishes, and using the $p$-theta law as appropriate.

Remark 4.7. The relation between $A$ and $B$ becomes, via the usual expression for the inverse, the expression

$$
\left(\begin{array}{cc}
0 & -1 \\
1 & 0
\end{array}\right) \tilde{B}_{n}\left(1 / q z ; v, w ; v^{\prime}, w^{\prime}\right)^{t}\left(\begin{array}{cc}
0 & 1 \\
-1 & 0
\end{array}\right) \tilde{B}_{n}\left(z ; v, w ; v^{\prime}, w^{\prime}\right)=C \tilde{A}_{n}(z ; v, w),
$$

where

$$
C=\frac{-q^{-1 / 2} I I_{n}\left(u_{0}^{\prime}, \ldots, u_{2 m+5}^{\prime}\right) G_{n}^{+}\left(v^{\prime}, w^{\prime}\right)}{I I_{n}\left(u_{0}, \ldots, u_{2 m+5}\right) F_{n}^{+}(v, w)} .
$$

Remark 4.8. In the form with apparent singularities, $B_{n}$ gets multiplied by $\theta_{p}(x z) / \theta_{p}\left(q^{n} x z\right)$, making it elliptic, as expected. Moreover, since for each generator of our lattice of shifts we have exhibited an isomonodromy transformation with coefficients of degree independent of $n$, the same holds for an arbitrary shift.

Once again, the special values, together with the determinant and the $p$-theta law, are more than sufficient to determine $\tilde{B}_{n}$; indeed, for generic parameters, any $m+3$ of the special values suffice. For both $\tilde{A}_{n}$ and $\tilde{B}_{n}$, this gives rise to a number of relations between the coefficients. For instance, using $\tilde{B}_{n}$, we can express

$$
G_{n}\left(u_{m+3} / q ; v^{\prime}\right) F_{n}^{+}\left(u_{m+3} / q, w\right)
$$

as an explicit linear combination of the terms

$$
G_{n}^{+}\left(u_{s} / q, v^{\prime}\right) F_{n}\left(u_{s} ; w\right)
$$

for $0 \leq s<m+3$. If we choose $v^{\prime}$ and $w$ suitably, we can arrange both for some terms of the resulting identity to drop out, and for the remaining integrals to be order $m$ elliptic Selberg integrals. For instance, if $v^{\prime}=u_{0} / q, w=\widehat{u_{1}}$, then

$$
G_{n}^{+}\left(u_{0} / q, v^{\prime}\right)=F_{n}\left(u_{1} ; w\right)=0,
$$

and thus the $s=0,1$ terms disappear, and we are left with an expression for

$$
G_{n}\left(u_{m+3} / q ; u_{0} / q\right) F_{n}\left(u_{1} ; u_{m+3} / q\right)
$$


as a linear combination of

$$
G_{n}^{+}\left(u_{s} / q, u_{0} / q\right) F_{n}^{-}\left(u_{s}, u_{1}\right)
$$

for $2 \leq s<m+3$. When $m=1$, these identities and the corresponding identities arising from the 12 entry of $A_{n}$ give direct proofs (i.e., without using the $E_{7}$ symmetry) of new special cases of Theorem 5.1 of [18] (which states that the elliptic Selberg integral satisfies bilinear relations making it a tau function for the elliptic Painlevé equation).

The special case $\tilde{B}\left(z ; u_{6} / q, u_{7} / q ; u_{0}, u_{1}\right)$ (with $m=1$ ) is particularly nice. In that case, in contrast to the situation with $\tilde{A}$, all of the integrals that appear in the expressions for the singular values are order 1 elliptic Selberg integrals, and may thus be expressed via (3.1) as tau functions for elliptic Painlevé. It should follow from a suitable Zariski density argument that the various formulas resulting from consistency of this expression and of the action of isomonodromy transformations continue to hold for arbitrary tau functions, with any appearance of $q^{n}$ replaced by $Q \in \mathbb{C}^{*}$ such that

$$
Q^{2} q^{-2} \prod_{0 \leq r<2 m+6} u_{r}=(p q)^{m+1} .
$$

(Sketch: As $n$ varies over (large) positive integers, the balancing condition describes a dense countable family of hypersurfaces in parameter space; that the contour integral is dense among all solutions on such hypersurfaces follows from the fact that its difference Galois group is generically equal to $\mathrm{GL}_{2}$.) However, since the arguments of [3] give a much more conceptual proof of this fact (and, conversely, that any function satisfying all consistency conditions is a tau function), there seems little point to fleshing out the details of a Zariski density argument.

Also of interest in the case $m=1$ is the relation to the action of the Weyl group $E_{7}$. This turns out to be easiest to describe in terms of the $B$ matrices, although what meaning this has from a difference equation perspective is as yet unclear.

Theorem 4.9. In the case $m=1$, let

$$
u_{r}^{\prime}= \begin{cases}q^{-1 / 2} u_{r} / x, & 0 \leq r<4 \\ q^{1 / 2} u_{r} x, & 4 \leq r<8\end{cases}
$$

where

$$
x=\left(\frac{u_{0} u_{1} u_{2} u_{3}}{u_{4} u_{5} u_{6} u_{7}}\right)^{1 / 4}=\left(\frac{p q^{2-n}}{u_{4} u_{5} u_{6} u_{7}}\right)^{1 / 2}=\left(\frac{u_{0} u_{1} u_{2} u_{3}}{p q^{2-n}}\right)^{1 / 2} .
$$

Then there exist matrices $C$ and $D$ independent of $z$ such that

$$
B_{n}\left(z ; v, w ; v^{\prime}, w^{\prime} ; u_{0}, \ldots, u_{7}\right)=C B_{n}\left(q^{1 / 2} x z ; v^{\prime \prime}, w^{\prime \prime} ; v^{\prime \prime \prime}, w^{\prime \prime \prime} ; u_{0}^{\prime}, \ldots, u_{7}^{\prime}\right) D .
$$

Proof. Indeed, it suffices to verify this in the special case

$$
\begin{array}{llll}
v=u_{6} / q, & w=u_{7} / q, & v^{\prime}=u_{0} / q, & w^{\prime}=u_{1} / q, \\
v^{\prime \prime}=u_{6}^{\prime} / q, & w^{\prime \prime}=u_{7}^{\prime} / q, & v^{\prime \prime \prime}=u_{0}^{\prime} / q, & w^{\prime \prime \prime}=u_{1}^{\prime} / q,
\end{array}
$$

for $z=u_{r} / q, 4 \leq r \leq 7$, in which case it follows readily by several applications of the transformation law [19, Theorem 9.7]

$$
I I_{n}\left(u_{0}, \ldots, u_{7}\right)=\prod_{1 \leq j \leq n}\left(\prod_{0 \leq r<s<4} \Gamma_{p, q}\left(q^{n-j} u_{r} u_{s}\right) \prod_{4 \leq r<s<8} \Gamma_{p, q}\left(q^{n-j} u_{r} u_{s}\right)\right) I I_{n}\left(u_{0}^{\prime}, \ldots, u_{7}^{\prime}\right)
$$

Finally, we observe that the case $m=0$ is precisely the elliptic hypergeometric equation [24] for a (terminating) elliptic hypergeometric series, since in that case the biorthogonal functions are Spiridonov's elliptic hypergeometric biorthogonal functions. 


\section{Acknowledgements}

The author would like to thank N. Witte for some helpful discussions of the orthogonal polynomial approach to isomonodromy (and the University of Melbourne for hosting the author's sabbatical when the discussions took place), and D. Arinkin and A. Borodin for discussions leading to [3] (and thus clarifying what needed (and, perhaps more importantly, what did not need) to be established here). The author was supported in part by NSF grant numbered DMS0401387, with additional work on the project supported by NSF grants numbered DMS-0833464 and DMS-1001645.

\section{References}

[1] Adler M., van Moerbeke P., The spectrum of coupled random matrices, Ann. of Math. (2) 149 (1999), 921-976, hep-th/9907213.

[2] Arinkin D., Borodin A., Moduli spaces of $d$-connections and difference Painlevé equations, Duke Math. J. 134 (2006), 515-556, math.AG/0411584.

[3] Arinkin D., Borodin A., Rains E.M., in preparation.

[4] Birkhoff G.D., General theory of linear difference equations, Trans. Amer. Math. Soc. 12 (1911), $243-284$.

[5] Borodin A., Isomonodromy transformations of linear systems of difference equations, Ann. of Math. (2) 160 (2004), 1141-1182, math.CA/0209144.

[6] Deift P.A., Orthogonal polynomials and random matrices: a Riemann-Hilbert approach, Courant Lecture Notes in Mathematics, Vol. 3, New York University, Courant Institute of Mathematical Sciences, New York, 1999.

[7] van Diejen J.F., Spiridonov V.P., An elliptic Macdonald-Morris conjecture and multiple modular hypergeometric sums, Math. Res. Lett. 7 (2000), 729-746.

[8] Etingof P.I., Difference equations with elliptic coefficients and quantum affine algebras, hep-th/9312057.

[9] Etingof P.I., Galois groups and connection matrices of q-difference equations, Electron. Res. Announc. Amer. Math. Soc. 1 (1995), no. 1, 1-9.

[10] Fokas A.S., Its A.R., Kitaev A.V., The isomonodromy approach to matrix models in 2D quantum gravity, Comm. Math. Phys. 147 (1992), 395-430.

[11] Forrester P.J., Witte N.S., Bi-orthogonal polynomials on the unit circle, regular semi-classical weights and integrable systems, Constr. Approx. 24 (2006), 201-237, math.CA/0412394.

[12] Kajiwara K., Masuda T., Noumi M., Ohta Y., Yamada Y., ${ }_{10} E_{9}$ solution to the elliptic Painlevé equation, J. Phys. A: Math. Gen. 36 (2003), L263-L272, nlin.SI/0303032.

[13] Kitaev A.V., Special functions of the isomonodromy type, Acta Appl. Math. 64 (2000), 1-32.

[14] Krichever I.M., Analytic theory of difference equations with rational and elliptic coefficients and the Riemann-Hilbert problem, Uspekhi Mat. Nauk 59 (2004), no. 6, 111-150 (English transl.: Russian Math. Surveys 59 (2004), no. 6, 1117-1154), math-ph/0407018.

[15] Magnus A.P., Painlevé-type differential equations for the recurrence coefficients of semi-classical orthogonal polynomials, J. Comput. Appl. Math. 57 (1995), 215-237, math.CA/9307218.

[16] Praagman C., Fundamental solutions for meromorphic linear difference equations in the complex plane, and related problems, J. Reine Angew. Math. 369 (1986), 101-109.

[17] van der Put M., Singer M.F., Galois theory of difference equations, Lecture Notes in Mathematics, Vol. 1666, Springer-Verlag, Berlin, 1997.

[18] Rains E.M., Recurrences of elliptic hypergeometric integrals, in Elliptic Integrable Systems, Editors M. Noumi and K. Takasaki, Rokko Lectures in Mathematics, Vol. 18, Kobe University, 2005, 183-199, math.CA/0504285.

[19] Rains E.M., Transformations of elliptic hypergeometric integrals, Ann. of Math. (2) 171 (2010), 169-243, math.QA/0309252.

[20] Ruijsenaars S.N.M., A generalized hypergeometric function satisfying four analytic difference equations of Askey-Wilson type, Comm. Math. Phys. 206 (1999), 639-690. 
[21] Sakai H., Rational surfaces associated with affine root systems and geometry of the Painlevé equations, Comm. Math. Phys. 220 (2001), 165-229.

[22] Spiridonov V.P., Elliptic beta integrals and special functions of hypergeometric type, in Integrable Structures of Exactly Solvable Two-Dimensional Models of Quantum Field Theory (Kiev, 2000), NATO Sci. Ser. II Math. Phys. Chem., Vol. 35, Kluwer Acad. Publ., Dordrecht, 2001, 305-313.

[23] Spiridonov V.P., On the elliptic beta function, Uspekhi Mat. Nauk 56 (2001), no. 1, 181-182 (English transl.: Russian Math. Surveys 56 (2001), no. 1, 185-186).

[24] Spiridonov V.P., Classical elliptic hypergeometric functions and their applications, in Elliptic Integrable Systems, Editors M. Noumi and K. Takasaki, Rokko Lectures in Mathematics, Vol. 18, Kobe University, 2005, 253-288, math.CA/0511579.

[25] Szegö G., Orthogonal polynomials, American Mathematical Society Colloquium Publications, Vol. 23, American Mathematical Society, New York, 1939.

[26] Yamada Y., A Lax formalism for the elliptic difference Painlevé equation, SIGMA 5 (2009), 042, 15 pages, arXiv:0811.1796. 